\title{
Heart Rate Synchrony in Psychological Counseling: A Case Study
}

\author{
Kentaro Kodama ${ }^{1}$, Shintaro Tanaka ${ }^{2}$, Daichi Shimizu ${ }^{3}$, Kyoko Hori ${ }^{4}$, Hiroshi Matsui ${ }^{5}$ \\ ${ }^{1}$ Department of Economics, Kanagawa University, Kanagawa, Japan \\ ${ }^{2}$ Uehiro Research Division for Philosophy of Co-Existence, The University of Tokyo Center for Philosophy (UTCP), \\ Tokyo, Japan \\ ${ }^{3}$ Faculty of Education, The University of Tokyo, Tokyo, Japan \\ ${ }^{4}$ Department of Psychology and Social Welfare, Seigakuin University, Saitama, Japan \\ ${ }^{5}$ Faculty of Science and Engineering, Waseda University, Tokyo, Japan \\ Email: *kkodama@jindai.jp
}

How to cite this paper: Kodama, K., Tanaka, S., Shimizu, D., Hori, K., \& Matsui, H. (2018). Heart Rate Synchrony in Psychological Counseling: A Case Study. Psychology, 9, 1858-1874.

https://doi.org/10.4236/psych.2018.97108

Received: May 31, 2018

Accepted: July 22, 2018

Published: July 25, 2018

Copyright (C) 2018 by authors and Scientific Research Publishing Inc. This work is licensed under the Creative Commons Attribution International License (CC BY 4.0).

http://creativecommons.org/licenses/by/4.0/

\section{Abstract}

The present study examined interpersonal synchrony during psychological counseling, focusing on heart rate synchrony. In psychological counseling and psychotherapy, embodied synchrony is considered an important factor related to building rapport and empathy. Recent interpersonal synchrony/coordination studies have addressed this issue, not only at the behavioral level but also at the neurological (brain activity) and physiological (cardiac activity) levels. However, there is little known literature on heart rate synchrony in a psychological counseling context. Therefore, we conducted a single exploratory case study to ascertain whether heart rate synchrony was observed in a counseling session and how it related to therapeutic processes and psychological issues. One male university student and one male clinical psychologist participated in our experiment. The student had a counseling session for 50 minutes. Video data were recorded and two wearable sensors were attached to the chests of both participants to collect heart rate data. We applied nonlinear time series analyses, based on a recurrence analysis, to the heart rate data to quantitatively assess heart rate synchrony. A qualitative analysis was also conducted by three clinical psychologists, based on video data from the viewpoints of clinical psychology and psychotherapy. The results show that the heart rate synchrony between client and therapist was observed and changed dynamically during the session. The present study suggests that heart rate synchrony may occur in some clinically important scenes and reflect psychological factors (e.g., building rapport and empathy) and social relationships (e.g., leader-follower). The present study shows the applicability of recurrence-based analyses to complex heart rate data during psychological counseling, as ex- 
plored in other interpersonal synchrony studies. Further examinations using more data from multiple viewpoints are expected to support our findings and cast light on the relationship between embodied synchrony and psychological issues in the context of psychological counseling and psychotherapy.

\section{Keywords}

Psychotherapy, Embodiment, Interpersonal Coordination, Heart Rate, Recurrence Analysis

\section{Introduction}

One definition of synchrony is the dynamic and reciprocal adaptation of the temporal structure of behaviors between interactive partners (Delaherche et al., 2012). This type of interactive and interpersonal synchrony/coordination has been investigated within various research fields, including social psychology, clinical psychology, cognitive science, robotics (e.g., Bernieri \& Rosenthal, 1991; Keller, Novembre, \& Hove, 2014; Koole \& Tschacher, 2016; Schmidt \& Richardson, 2008; Wachsmuth, Lenzen, \& Knoblich, 2008). It is said that interpersonal synchrony/coordination is associated with psychological factors and social functions such as building rapport or trust (e.g., Bernieri, 1988; Miles, Nind, \& Macrae, 2009). It facilitates communication and cooperation and is also linked to psychological factors such as closeness, affiliation, and empathy (e.g., Hove \& Risen, 2009; Vicaria \& Dickens, 2016; Wiltermuth \& Heath, 2008). Recently, interpersonal synchrony/coordination has been observed not only in body movements, but also in brain activities (e.g., Dumas, Nadel, Soussignan, Martinerie, \& Garnero, 2010; Tognoli, Lagarde, DeGuzman, \& Kelso, 2007) and cardiac activities (e.g., Konvalinka et al., 2011; Mitkidis, McGraw, Roepstorff, \& Wallot, 2015). One of the interesting points is that since brain and cardiac activities cannot be voluntarily controlled, these synchronized phenomena emerge spontaneously. These activities also show complex and interesting features in their time series, because they can be considered a self-organizing system having nonlinear interactions among their components at multiple time scales (e.g., Kelso, 1995; Richardson \& Chemero, 2014; Sleimen-Malkoun, Temprado, \& Hong, 2014).

In psychotherapy studies, interpersonal synchrony/coordination has been seen as an important and relevant aspect of therapeutic skills (Tschacher \& Bergomi, 2011; Tschacher \& Pfammatter, 2016). Researchers have argued that nonverbal synchrony is associated with session-level processes and therapy outcomes (Ramseyer \& Tschacher, 2011) and that embodied synchrony can act as an indicator, reflecting the mental process of a client or counselor (Nagaoka, Yoshikawa, \& Komori, 2006). These previous studies have applied quantification methods (e.g., image-processing techniques) to video data to measure interpersonal bodily synchrony/coordination, using objective evidence to reveal the im- 
portance of such embodied interpersonal synchrony/coordination in psychotherapy (for reviews, Koole \& Tschacher, 2016; Ramseyer \& Tschacher, 2006). We therefore assume that interpersonal bodily synchrony/coordination reflects psychological processes and social relationships. In particular, the current study focuses on interpersonal synchrony at the physiological level (i.e., heart rate synchrony). Because it is hard to control cardiac activity voluntarily or to intend to synchronize with others, we suppose that it can be an objective measure for psychological counseling.

In recent years, some researchers have examined interpersonal heart rate synchrony. Konvalinka and colleagues revealed the physiological effects of synchronized arousal in a collective ritual between active participants and related spectators, but not between participants and other members of the audience (Konvalinka et al., 2011). They suggested that a collective ritual may evoke synchronized arousal between active participants and bystanders over time-and that relatedness may play a role in structuring people's heart rates with those close to them (Konvalinka et al., 2011). Mitkidis and colleagues investigated whether trust has an effect on heart rate synchrony using an economic game (Mitkidis et al., 2015). They found that the degree of heart rate synchrony is predictive of participants' expectations regarding their partners in the economic game, concluding that the observed physiological and behavioral changes are indications of a trust-building process (Mitkidis et al., 2015). These earlier studies have shown that heart rate synchrony can be an objective measure reflecting psychological and social factors.

We view heart rate synchrony as an objective measure that may reflect social relationships and psychological processes in psychological counseling. Objective and quantitative assessments of psychological counseling/treatment and psychotherapy have been the focus of much attention and interest (e.g., Sales \& Alves, 2016). Although some research groups have applied assessments based on heart rate variability analyses to clinical psychology and psychotherapy (e.g., Kiema, Rantanen, Laukka, Siipo, \& Soini, 2014), these have measured the heart rates of clients only, not those of both clients and therapists. A survey of the literature suggests that previous studies have not measured the heart rates of both parties; there is little known literature about the heart rate synchrony in a psychological counseling context. Thus, the present study investigates the heart rate synchrony between a client and a therapist during psychological counseling. Because it is the first exploratory study to observe the heart rate synchrony phenomenon in a psychological counseling context and because it obtains data for generating hypotheses which should be examined statistically and scientifically in the future experiments, we begin with a single case study.

\section{Method}

\subsection{Participants}

One male university student (21 years old) and one male clinical psychologist (30 years old) participated in our experiment. The student was recruited as a 
client, while the therapist was one of the coauthors, a professional with more than five years of clinical experience. The client underwent a counseling session lasting 50 minutes. The therapist considered the client to be a healthy male student without a specific mental disorder, because it seemed that the client did not need clinical treatment for any dysphoria or anxiousness. After setting the agenda, the client discussed his difficult situation (i.e., interpersonal relationships in his university activities) rather than his personal mental health issues in detail. However, another issue that underpinned his difficult situation and affected his emotional state (i.e., male-female relationships) was revealed during the latter part of the session.

The procedures were approved by the research ethics committee of Kanagawa University, where the experiment was conducted. Each participant provided written informed consent before being allowed to participate in this study. The student was paid 1,000 JPN yen per hour for his participation.

\subsection{Apparatus}

The participants were seated 1.5 meters apart, facing each other. A video camera (Handycam HDR-PJ390, Sony) and two microphones (4071 Miniature Omnidirectional Microphone, DPA microphones) were used to record and collect audio-visual data. The two microphones were separately connected to the audio mixer (R-26 Portable Recorder, Roland). The left and right microphones were used by the client and therapist, respectively. Cardiac activities were measured using a wearable sensor (MyBeat, Union tools Inc.), attached to the chest of each participant. This sensor can export the RR intervals (RRI) time series as raw data. For the data processing and analysis, a personal computer (LET'S NOTE CF-SX2, Panasonic), software (MATLAB R2014a, MathWorks) (R, 3.1.2), and programs (Cross Recurrence Plot Toolbox for $\mathrm{MATLAB}^{\circledR}$, Ver. 5.22 (R32.2); Marwan, Carmen Romano, Thiel, \& Kurths, 2007) (R “crqa" package, Ver. 1.0.6; Coco \& Dale 2014) were used.

\subsection{Procedure: Data Collection and Analyses}

In the experimental room (four meters square), a video camera was positioned at a distance of three meters from the participants. Wearable sensors were attached to the chests of both participants individually to collect heart rate data before they entered the room. The client was seated and waiting in the room before the therapist entered. The client was asked to undergo psychological counseling for 50 minutes. The experimenter told the therapist that the client was a male university student. He was not informed of the client's problems before the session. When the therapist entered the room, he sat in the chair facing the client. This was the first contact between them. The experimenter left the room a few minutes after attaching microphones to the participants and beginning to record audio-visual data. The counseling session then began; it was videotaped for about 50 minutes, using a camera. After completing the session, the therapist 
called the experimenter into the room to stop the recording.

The video data were qualitatively analyzed by three clinical psychologists (including the therapist who participated in the experiment). They analyzed the session from the viewpoints of clinical psychology and psychotherapy, dividing it into several phases to describe the changing counseling process. Categorization was performed from the viewpoint of clinical meaning and function. The results of the qualitative analysis were integrated with those of the quantitative analysis (i.e., the time series analysis of heart rate data).

To convert the raw RRI time series data into beat-per-minute (BPM) data before the time series analysis, the RRI time series was averaged over non-overlapping intervals of 1 and $10 \mathrm{~s}$ for the nonlinear time series analysis (i.e., recurrence analysis) and for producing an observation of the overall tendency of the two sets of time series data, respectively. Because converting to BPM data means normalizing and smoothing the raw RRI data-the shorter the intervals for averaging RRI data, the weaker the smoothing (Wallot, Fusaroli, Tylén, \& Jegindø, 2013) - we used BPM data averaged by $1 \mathrm{~s}$ intervals (relatively preserving the original variability of the heart rate activity) for the nonlinear time series analysis and BPM data averaged by $10 \mathrm{~s}$ intervals for the qualitative observation (it was relatively easy to visually identify the time-varying structure of the data).

To capture and quantify the interpersonal dynamics between participants, we used a nonlinear time series analysis, based on a recurrence analysis (for a tutorial, Webber \& Zbilut, 2005), which was originally developed to uncover subtle temporal correlations and repetitive patterns. It is relatively free of assumptions about data size and distribution (Zbilut \& Webber, 1992). Time series data is embedded and its trajectory is reconstructed in a higher dimensional phase space. The distances between all possible combinations of each vector are calculated and distributed within a distance matrix (Webber \& Zbilut, 2005). All elements in the distance matrix with distances at or below the threshold (i.e., radius) are defined as recurrent (recurrence points) and included in the recurrence matrix, while all other elements are excluded from it (Webber \& Zbilut, 2005). Such calculations and definitions are used to construct a recurrence plot (RP), which is a method of visualization that shows the dynamic properties and temporal patterns of the system as a two-dimensional representation (Eckmann, Kamphorst, \& Ruelle, 1987). A recurrence quantification analysis (RQA) allows researchers to quantify and assess the dynamic structures and properties of a dynamical system, based on RP or phase space trajectory (more detail in Webber \& Zbilut, 2005).

A cross recurrence plot (CRP) and cross recurrence quantification analysis (CRQA) are versions of recurrence analysis (i.e., RP and RQA) applied to two time series (Zbilut, Giuliani, \& Webber, 1998). They capture the synchronizing/coordinating properties that result from two streams of information interacting over time (Coco \& Dale, 2014), in other words, quantifying the extent to which two observed data series unfold in similar ways over time (Shockley, 2005). To measure interpersonal synchrony/coordination, two CRQA measures 
are regarded as significant indices. First, the percentage of recurrence (\%Recurrence) corresponds to the ratio of the number of shared locations, relative to the number of possible shared locations in the phase space (Shockley, 2005). In other words, \%Recurrence reflects shared locations in the phase space and captures the overall synchrony state and the coordination stability between two systems; in the present study, \%Recurrence is viewed as an index of how often the two systems synchronize. Second, the percentage of determinism (\%Determinism) indicates the percentage of recurrence points forming diagonal lines in the RP, given a minimal length threshold (Coco \& Dale, 2014). \%Determinism captures the way in which a system is organized in terms of similar sequences of values that can change over time (Wallot, Mitkidis, McGraw, \& Roepstorff, 2016). It can be interpreted as continued synchrony/coordination over time; in this study, \%Determinism is regarded as an index of how long synchrony persists between two systems.

CRP and CRQA have been widely applied in interpersonal synchrony/coordination studies (for reviews, Fusaroli, Konvalinka, \& Wallot, 2014; Shockley \& Riley, 2015), from the behavioral to the physiological level, including heart rate synchrony (Konvalinka et al., 2011; Mitkidis et al., 2015; Wallot et al., 2016). The present study applies them to the heart rate data of a client and therapist during a psychological counseling session to describe the dynamically changing process of an interpersonal relationship, focusing on heart rate synchrony. We performed CRP and CRQA using the Cross Recurrence Plot Toolbox for MATLAB $^{\oplus}$, Ver. 5.22 (R32.2) (Marwan et al., 2007) and the R package “crqa” (version 1.0.6) (Coco \& Dale, 2014), after determining the optimal values for the input parameters (e.g., time delay, embedding dimension, radius), referring to the standard RQA method guidelines (Webber \& Zbilut, 2005). We chose time delay, embedding dimension, and radius in phase space (after rescaling by mean distance and z-score normalization) for each analysis, $(6,5$, and 40 , respectively). We also conducted a windowed CRQA to investigate CRQA measures in the moment by shifting the window step by step (Coco \& Dale, 2014). The window size was 300 points ( 5 minutes) based on a reference to the setting in a previous study (Konvalinka et al., 2011). We shifted the window with a 60-point (1 minute) step. Finally, we quantified the (a) symmetry property of CRP to assess the leader-follower relationship during the counseling session overall and in each phase. Using a diagonal-based analysis (Coco \& Dale, 2014; Shockley \& Riley, 2015), we calculated the ratio of recurrence points in the upper/lower triangular region of the CRP to the total recurrence points, excluding the main diagonal line. If the recurrence point ratios of the upper and lower triangular regions were equal, the ratio would 50\%. The upper/lower triangular region corresponds to an earlier point in time for one than another (Shockley \& Riley, 2015). We assumed that if the ratio was higher than 50\% (greater recurrence than another region), this would mean that the higher ratio in triangular region of the CRP was the leader. 


\section{Results and Discussion}

\subsection{Summary of the Session}

The client was a healthy male student without any specific depression or anxiety. At the beginning of the session, after a brief self-introduction and ice breaker, the client and therapist decided the session agenda, what the client wanted to talk about, and how to use the counseling time. The client discussed a difficult situation rather than mental health issues in detail. In particular, he discussed a personal relationship and organizational operations in his university activities. The therapist continued to listen to the client, focusing mainly on his difficult situation and behavior in trying to resolve it. The therapist provided a supportive counseling approach from the viewpoint of solution-focused brief therapy (Franklin, 2012). Throughout the counseling session, the client changed his viewpoint and position. At the beginning of the session, he believed that his difficult situation was caused by difficult interpersonal communication. However, he changed his position and enriched his understanding, accepting that his problems might have been caused by his own way of communicating with others, particularly female students. Thus, during the latter part of the session, the therapist had an insight into another issue (i.e., male-female relationships) underpinning the client's difficult situation.

After the counseling session, a qualitative analysis based on the video data was conducted to categorize the session in terms of clinical meaning and function. Three clinical psychologists, including the therapist who participated in the experiment, observed the video, discussed the session, and categorized it into the following three phases: 1) Setting phase. At the beginning of the session, the therapist confirmed the mental health status of the client through a brief conversation about his daily life and recent history. This conversation seemed to relax the client (i.e., it served as the ice breaker). Next, the therapist asked the client about the main topic, his current problems (i.e., the agenda setting). The client mentioned two issues, his job search and university activities; the latter issue was selected. 2) Advising phase. The therapist listened carefully to the client and collected information to develop a hypothesis about the client's core issue. The hypothesis was that the basis of the issue was that the client faced difficulty in communicating interactively with others. For example, he could convey his intention to others only unidirectionally not bidirectionally; otherwise he could only wait for others to change passively, but he could not change others actively. After coming up with a hypothesis, the therapist gave some advice, which the client accepted. 3) Re-finding phase. The topic changed and the therapist discovered another issue about male-female relationships and the client's love affair. The client himself re-found the difficulty he experienced in communicating with female students (Table 1).

\subsection{Raw data: RRI and BPM}

As shown in the raw RRI data (Figure 1, upper panel), the RRI appeared to be longer for the therapist than for the client; in other words, the BPM was slower 
Table 1. Categorization of the phases of the counseling session.

\begin{tabular}{cccc}
\hline Phase & Time $[\mathrm{mm}: \mathrm{ss}]$ & Duration $[\mathrm{s}]$ & Content \\
\hline 1 & $0: 00-5: 30$ & 330 & $\begin{array}{c}\text { Setting: Agenda and goal setting were established. } \\
2\end{array}$ \\
$5: 30-38: 10$ & 1960 & $\begin{array}{c}\text { Advising: The therapist advised the client. } \\
\text { Re-finding: Another issue involving interpersonal } \\
\text { communication was found. }\end{array}$ \\
\hline
\end{tabular}

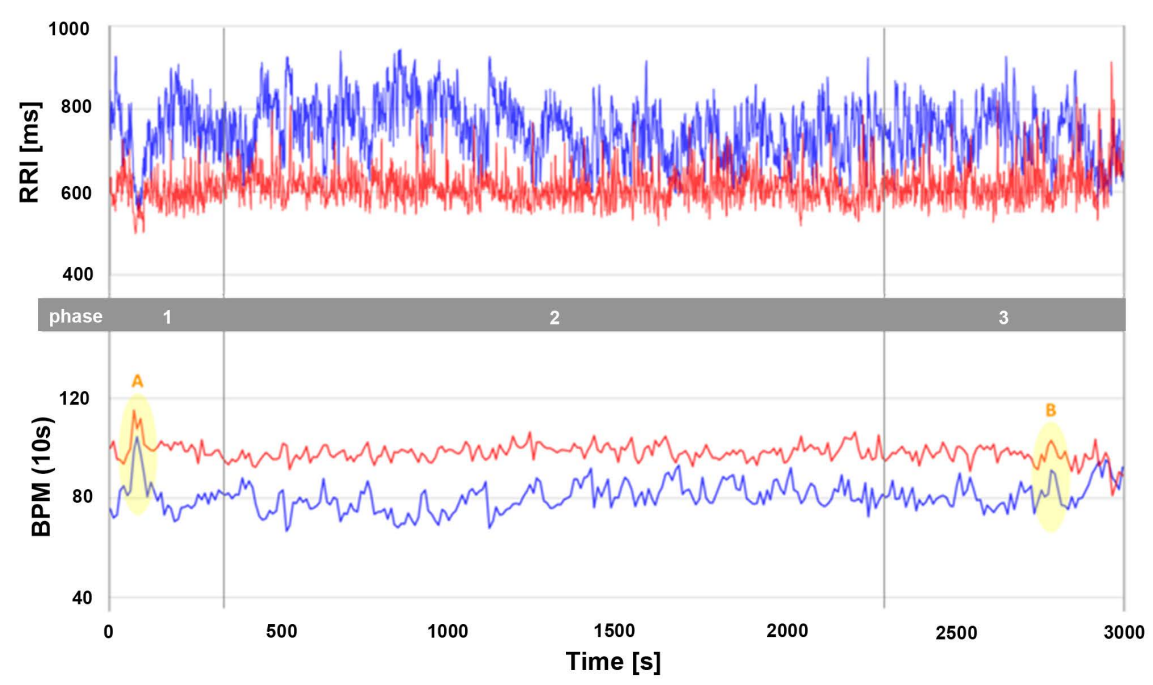

Figure 1. RR Intervals (RRI) [ms] and Beats-Per-Minute [BPM].

for the therapist than for the client, on average. Actually, the mean RRI of the therapist was $745.28(S D=68.56)[\mathrm{ms}]$, while that of the client was $610.51(S D=$ 39.53) [ms] during the session (Table 2). The mean BPM of the therapist was $80.54(S D=7.34)[\mathrm{BPM}]$, while that of the client was $98.29(S D=6.04)[\mathrm{BPM}]$ during the session. Throughout the session, although the therapist's BPM stayed constant, the client's BPM seemed to get slower (Table 2).

Because the RRI time series looked very complex and noisy, we calculated the BPM for normalizing and smoothing the raw RRI data by averaging the RRI time series over non-overlapping intervals of 10 seconds (Figure 1, lower panel). This enabled us to easily compare the BPM data of the client and therapist, finding characteristic scenes in which they seemed to co-vary. For example, in Scenes A and B, the client's BPM (red line) and the therapist's BPM (blue line) seemed to increase and decrease simultaneously. During Scene A (1:00 - 2:00), after the brief self-introduction, the therapist set the agenda about what the client wanted to discuss and the client began to answer questions. During Scene B (46:00 - 47:00), the therapist discovered that the client had another relevant issue involving male-female relationships and asked the client about his past relationships. Although these scenes were clinically important, it is not possible to say why or how such scenes affect interpersonal heart rate synchrony at the physiological level. Next, we show the result of the quantitative and objective assessment using recurrence analysis to complement the qualitative analyses. 
Table 2. RR intervals (RRI) and beats-per-minute (BPM) in each phase.

\begin{tabular}{ccccc}
\hline \multirow{2}{*}{ Phase } & \multicolumn{2}{c}{ Mean RRI (SD) $[\mathrm{ms}]$} & \multicolumn{2}{c}{ Mean BPM (SD) [BPM] } \\
\cline { 2 - 5 } & Client & Therapist & Client & Therapist \\
\hline 1 & $596.57(37.74)$ & $741.43(76.46)$ & $100.66(6.18)$ & $80.97(8.59)$ \\
2 & $609.73(36.33)$ & $750.18(67.53)$ & $98.41(5.59)$ & $80(7.10)$ \\
3 & $615.57(41.76)$ & $736.57(65.68)$ & $97.49(6.22)$ & $81.52(7.08)$ \\
Mean & $610.51(39.53)$ & $745.28(68.56)$ & $98.29(6.04)$ & $80.54(7.34)$ \\
\hline
\end{tabular}

\subsection{Cross-Recurrence Plots}

Figure 2 shows the CRP of the entire counseling session (top left-hand side), Phase 1 (top right-hand side), Phase 2 (bottom left-hand side), and Phase 3 (bottom right-hand side). The diagonal red line represents the line of temporal coincidence or synchrony, where the lag is zero and the heart rate of the client and therapist synchronize. The blue lines in the top left-hand panel represent the borders between the phases. The white bands can be interpreted as abrupt changes in the dynamics; they imply extreme events (Marwan et al., 2007). Throughout the session, we observed some structured patterns, rather than a random dots pattern. Moreover, the recurrence structures seemed to differ among the phases.

\subsection{Cross Recurrence Quantification Analysis}

To quantitatively confirm the results of qualitative observations of the CRP, a CRQA was conducted. Figure 3 shows the CRQA measures, \%Recurrence, and \%Determinism for the entire counseling session (grey bar) and for each phase (black bars). The \%Recurrence was $2.51 \%$ for the entire counseling session, 3.38\% in Phase 1, 2.30\% in Phase 2, and 1.94\% in Phase 3. The \%Determinism was $28.06 \%$ for the entire counseling session, $30.36 \%$ in Phase $1,26.29 \%$ in Phase 2, and 21.74\% in Phase 3.

As mentioned above, the \%Recurrence of CRQA can be interpreted as the overall stability of synchrony/coordination between two time series (how often two systems synchronize), while the \%Determinism of CRQA can be interpreted as continued synchrony/coordination over time (how long synchrony persists). As shown in Figure 3, both \%Recurrence and \%Determinism were higher in Phase 1 than in the other phases.

This means that heart rate synchrony increased between the client and therapist in the first phase of the session. Such a high level of synchrony between the participants' heart rates might indicate that rapport or trust was growing (Miles et al., 2009; Mitkidis et al., 2015). Because building rapport or trust is an important factor in psychotherapy and since interpersonal synchrony is supposed to reflect this (Ramseyer \& Tschacher, 2011), this result may show the outcome of therapeutic skill. Figure 3 also shows that both \%Recurrence and \%Determinism declined gradually throughout the session, from Phase 1 to 

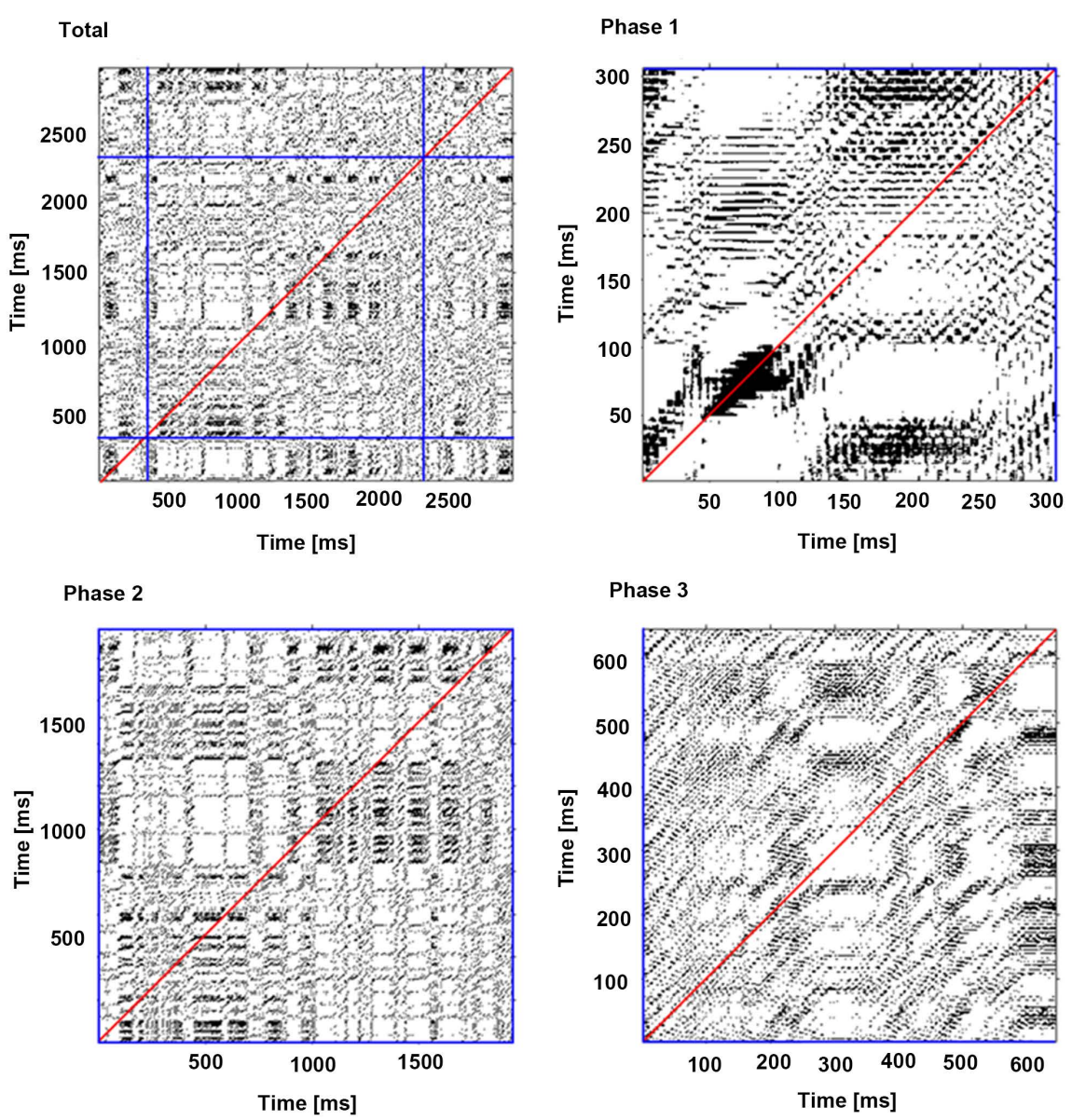

Figure 2. Cross recurrence plot (Top left-hand side: entire counseling session, Top right-hand side: Phase 1, Bottom left-hand side: Phase 2, Bottom right-hand side: Phase 3).

Phase 3. We speculate that the participants' heart rates did not always need to synchronize once trust or rapport existed. However, since the data lengths are different in different phases, we should analyze the dynamics in more detail during each phase to detect dynamic changes in the heart rate synchrony pattern.

Figure 4 presents the results of the windowed CRQA. Dynamic changes are shown in \%Recurrence (Figure 4, top panel) and \%Determinism (Figure 4, bottom panel) across each one-minute interval. In each panel, dashed lines indicate the mean value of each measure (\%Recurrence or \%Determinism), red circles represent the highest value in the session, and blue circles show values higher than the mean values. Both \%Recurrence and \%Determinism were at their highest during the initial phase (1:00 - 2:00), when the therapist set the agenda and the client briefly talked about his troubles briefly, as discussed in 3.1. It is worth asking why such a fast heart rate synchrony was observed so soon after the session began. The actual interaction between the client and therapist started while the experimenter was setting up the apparatus and explaining the experiment briefly $-a$ few minutes before the actual session began. Although the 

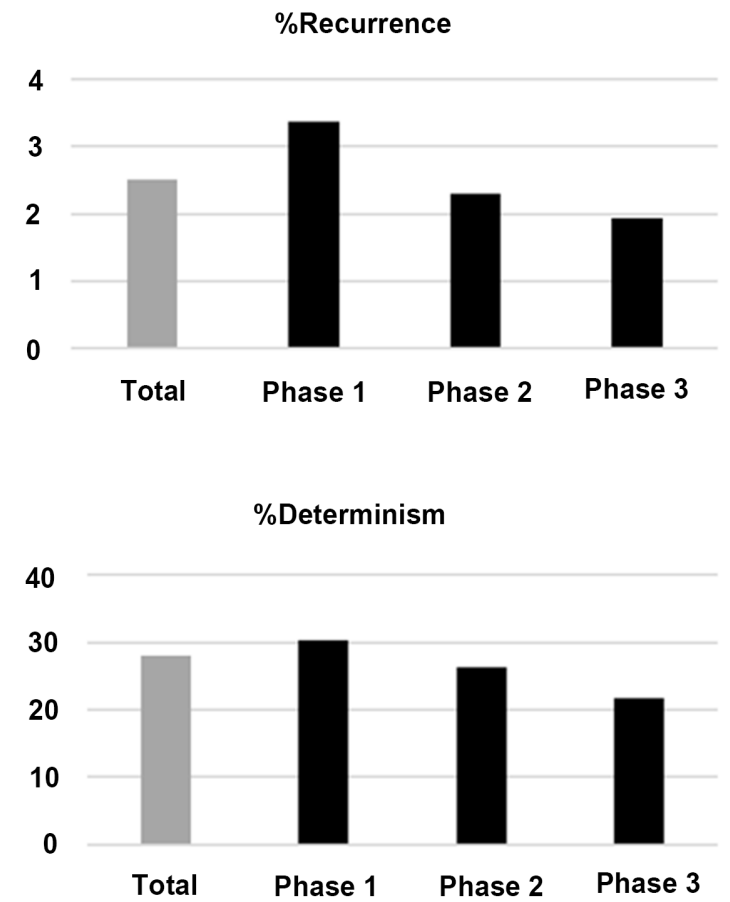

Figure 3. Cross recurrence quantification analysis measures (Top: \%Recurrence, Bottom: \%Determinism).

\section{\%Recurrence}

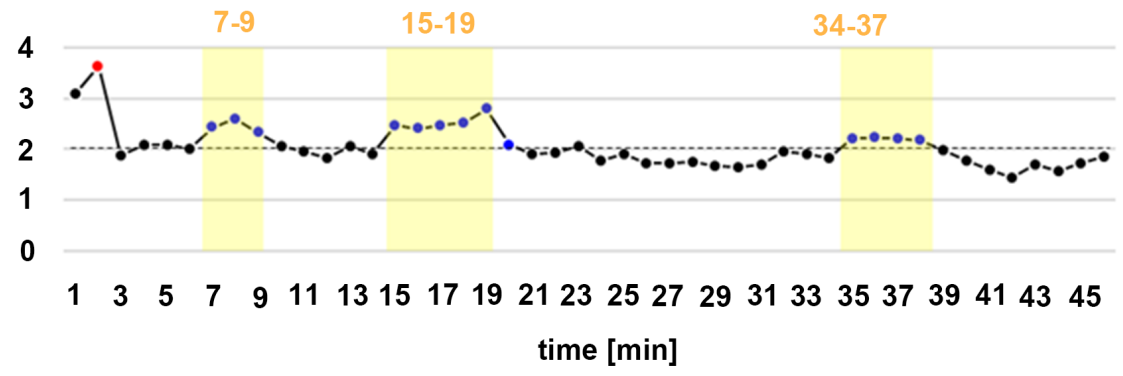

\section{\%Determinism}

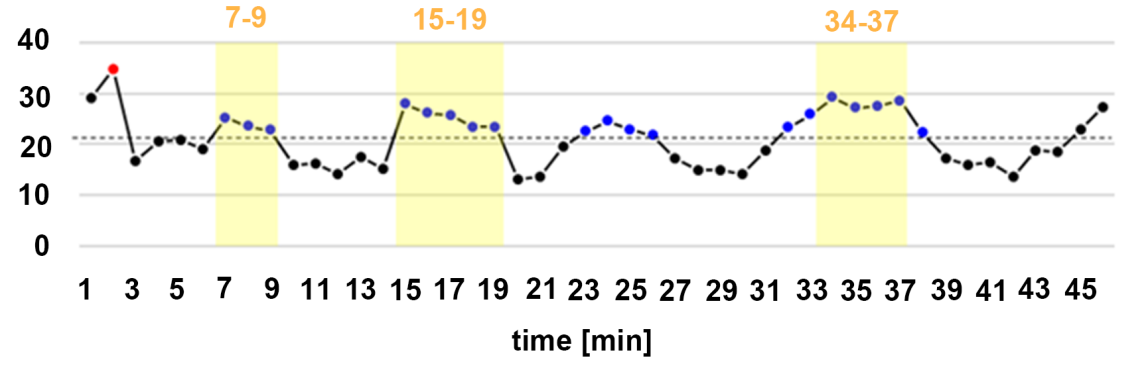

Figure 4. Dynamic change in the cross-recurrence quantification analysis measures.

reason was not clear, we inferred that this interaction might have affected the result. We focused on scenes in which both measures (\%Recurrence and \%Determinism) were higher than the mean values continuously-for longer than three minutes (the yellow bands in Figure 4). 
During the first scene (7:00 - 9:00) the client discussed a problem involving his university activities (e.g., how he could motivate group members and make them participate in activities). The therapist listened carefully to the client and asked him some questions regarding the details of the activity. In this scene, the therapist appeared to listen attentively, nodding. After the session, the therapist regarded this scene as the information-collecting phase, used to develop a hypothesis. In the second scene (15:00 - 19:00) the client talked about what he wanted (e.g., to have all members actively participate, including passive members). The therapist asked the client about his role in the group activity and whether participation was subjective or forced. The client said that he felt that participation was both subjective and forced. The therapist engaged in empathetic listening, appeared to locate the important issue underpinning the client's problem, and focused on his thoughts and feelings. In the third scene (34:00 - 37:00) another issue was discovered (i.e., male-female relationships). The client had a problem with interpersonal communication, particularly with female students. The client revealed that something was bothering him (e.g., how to behave when communicating with female students and how to overcome his hesitation about meeting them one-on-one). The therapist listened with empathy, encouraged the client, and clarified the issue. The client himself also seemed to feel that he now understood the cause of his problems.

Finally, we presented the leader-follower relationship across the entire counseling session and during each phase (Figure 5). The ratio of recurrence points in the upper/lower triangular region of CRP were calculated using a diagonal-based analysis. We assumed that if the ratio was higher than $50 \%$ (greater recurrence than another region), this would mean that the higher ratio in triangular region of the CRP was the leader. The current data suggest that the therapist/client is the leader/follower when the upper/lower triangular region ratio is higher than $50 \%$. Overall, the therapist tended to lead the client in terms of heart rate synchrony (55.69\% and $44.31 \%$, respectively). In Phase 1, the therapist's ratio (48.31\%) was slightly lower than the client's ratio (51.69\%), while in Phase 3, the therapist's ratio $(52.12 \%)$ was higher than the client's ratio $(47.88 \%)$. Although the ratio differences during these phases were subtle, interestingly, in Phase 2 the therapist's ratio (58.66\%) was relatively higher than the client's ratio (48.31\%). In this phase, the therapist actively questioned the client to collect information to develop a hypothesis about the client's problem. He then gave the client some advice about ways to resolve his difficult situation. The fact that the therapist tended to lead the client, in terms of heart rate synchrony, might suggest that the leader-follower relationship observed in the CRP reflected the therapist's behavior in collecting information and providing advice.

By integrating the recurrence-based quantitative analysis with the qualitative analysis conducted by therapists, it became suggestive that the scenes highlighted by the recurrence-based quantitative analysis shared a commonality: the therapist displayed empathy and listened attentively. Although the present finding has been derived from a counseling session in a single case, it suggests that 


\section{Ratio of recurrence on each side of CRP}

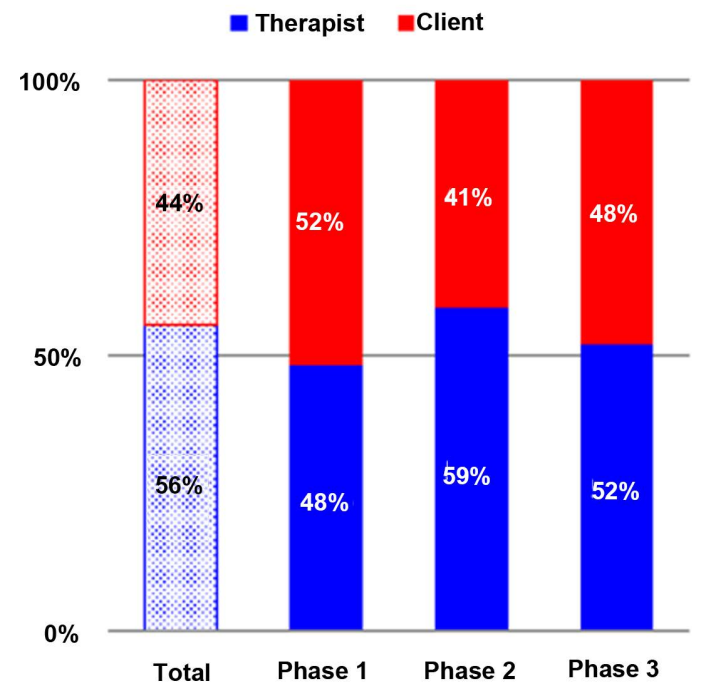

Figure 5. Ratio of Recurrence on Each Side of CRP.

when a therapist shows empathetic and attentive behavior, heart rate synchrony can occur.

\subsection{Future Directions}

We conducted a single case study as a first step to explore the relationship between physiological interaction (heart rate synchrony) and psychological issues in psychological counseling context. One of the limitations of our study is that we extracted data from only a single case. Therefore, for future studies, we aim to collect more data (a larger sample size) to confirm whether similar tendencies and dynamic patterns are found in other cases (e.g., initial high synchrony at the beginning of a session, a change in the leader-follower relationship during a session associated with a counseling process). Another limitation of our study is that our consideration is just a matter of speculation. If we obtain more data, we can statistically assess the relations and correlations between bodily synchrony (e.g., heart rate synchrony), session outcomes, and therapeutic skill, as previous studies have done (e.g., Ramseyer \& Tschacher, 2011, 2014; Tschacher \& Pfammatter, 2016). To achieve this, a quantitative assessment of the counseling session using existing tools (e.g., Sales \& Alves, 2016) should be combined with sensors or computer-based data processing techniques (e.g., Paxton \& Dale, 2012) to derive an objective measurement. Although the current study focused only on heart rate synchrony, a heart rate variability analysis, using a time- and frequency-domain to assess psychological stress and emotional factors (Appelhans \& Luecken, 2006) should be conducted to analyze the issue in more detail. Other measurements, such as nonverbal body synchrony (Nagaoka \& Komori, 2008; Ramseyer \& Tschacher, 2011) and vocal synchrony (Reich, Berman, \& Dale, 2014) should also be explored to understand multi-modal and multidimensional complex interactions, like psychological counseling. Moreover, a re- 
currence-based analysis should be applied to verbal data, such as texts or transcripts (Orsucci et al., 2006) and conceptual similarity (Angus, Watson, Smith, Gallois, \& Wiles, 2012). A multidimensional description including such verbal and conceptual levels could provide a deeper understanding of body-mind interplay during a counseling session and suggest some practical applications in clinical psychology and psychotherapy. Such applications could include reflection and feedback on counseling sessions for, and the training/education of, therapists. Simultaneously, it is also clinically important to investigate dynamic change across a relatively long span through longitudinal studies, because actual clinical counseling can rarely be completed in a single session.

\section{Conclusion}

The present study examined interpersonal synchrony during psychological counseling, focusing on heart rate synchrony. The results show that heart rate synchrony between client and therapist was observed and it dynamically changed during the session. It was also suggested that heart rate synchrony could be observed in some clinically important scenes and might reflect the social outcome (building rapport or trust) and relationship (leader-follower). The present study has shown that a recurrence-based analysis can be used to assess complex heart rate data during psychological counseling, as other interpersonal synchrony/coordination studies have done. Further studies using more data from multiple viewpoints are needed to support these findings and to expand the understanding of the relationship between embodied synchrony and psychological issues in the context of psychological counseling and psychotherapy.

\section{References}

Angus, D., Watson, B., Smith, A. E., Gallois, C., \& Wiles, J. (2012). Visualising Conversation Structure across Time: Insights into Effective Doctor-Patient Consultations. PLoS ONE, 7, e38014. https://doi.org/10.1371/journal.pone.0038014

Appelhans, B. M., \& Luecken, L. J. (2006). Heart Rate Variability as an Index of Regulated Emotional Responding. Review of General Psychology, 10, 229-240.

https://doi.org/10.1037/1089-2680.10.3.229

Bernieri, F. J. (1988). Coordinated Movement and Rapport in Teacher-Student Interactions. Journal of Nonverbal Behavior, 12, 120-138. https://doi.org/10.1007/BF00986930

Bernieri, F. J., \& Rosenthal, R. (1991). Interpersonal Coordination: Behavior Matching and Interactional Synchrony. In R. Feldman, \& B. Rimé (Eds.), Studies in Emotion \& Social Interaction. Fundamentals of Nonverbal Behavior (pp. 401-432). Cambridge: Cambridge University Press.

Coco, M. I., \& Dale, R. (2014). Cross-Recurrence Quantification Analysis of Categorical and Continuous Time Series: An R Package. Frontiers in Psychology, 5, 510.

https://doi.org/10.3389/fpsyg.2014.00510

Delaherche, E., Chetouani, M., Mahdhaoui, A., Saint-Georges, C., Viaux, S., \& Cohen, D. (2012). Interpersonal Synchrony: A Survey of Evaluation Methods across Disciplines. IEEE Transactions on Affective Computing, 3, 349-365.

https://doi.org/10.1109/T-AFFC.2012.12 
Dumas, G., Nadel, J., Soussignan, R., Martinerie, J., \& Garnero, L. (2010). Inter-Brain Synchronization during Social Interaction. PLOS ONE, 5, e12166.

https://doi.org/10.1371/journal.pone.0012166

Eckmann, J.-P., Kamphorst, S. O., \& Ruelle, D. (1987). Recurrence Plots of Dynamical Systems. Europhysics Letters (EPL), 4, 973-977.

https://doi.org/10.1209/0295-5075/4/9/004

Franklin, C. (2012). Solution-Focused Brief Therapy: A Handbook of Evidence-Based Practice. Oxford: Oxford University Press.

Fusaroli, R., Konvalinka, I., \& Wallot, S. (2014). Analyzing Social Interactions: The Promises and Challenges of Using Cross Recurrence Quantification Analysis (pp. 137-155). Cham: Springer. https://doi.org/10.1007/978-3-319-09531-8_9

Hove, M. J., \& Risen, J. L. (2009). It's All in the Timing: Interpersonal Synchrony Increases Affiliation. Social Cognition, 27, 949-960. https://doi.org/10.1521/soco.2009.27.6.949

Keller, P. E., Novembre, G., \& Hove, M. J. (2014). Rhythm in Joint Action: Psychological and Neurophysiological Mechanisms for Real-Time Interpersonal Coordination. Philosophical Transactions of the Royal Society of London. Series B, Biological Sciences, 369, 20130394. https://doi.org/10.1098/rstb.2013.0394

Kelso, J. A. S. (1995). Dynamic Patterns: The Self-Organization of Brain and Behavior. MIT Press.

Kiema, H., Rantanen, A., Laukka, S., Siipo, A., \& Soini, H. (2014). The Connection between Skilled Counseling and Client's Heart Rate Variability. Procedia-Social and Behavioral Sciences, 159, 802-807. https://doi.org/10.1016/j.sbspro.2014.12.452

Konvalinka, I., Xygalatas, D., Bulbulia, J., Schjodt, U., Jegindo, E.-M., Wallot, S., \& Roepstorff, A. (2011). Synchronized Arousal between Performers and Related Spectators in a Fire-Walking Ritual. Proceedings of the National Academy of Sciences, 108, 8514-8519. https://doi.org/10.1073/pnas.1016955108

Koole, S. L., \& Tschacher, W. (2016). Synchrony in Psychotherapy: A Review and an Integrative Framework for the Therapeutic Alliance. Frontiers in Psychology, 7, 1-17. https://doi.org/10.3389/fpsyg.2016.00862

Marwan, N., Carmen Romano, M., Thiel, M., \& Kurths, J. (2007). Recurrence Plots for the Analysis of Complex Systems. Physics Reports, 438, 237-329. https://doi.org/10.1016/j.physrep.2006.11.001

Miles, L. K., Nind, L. K., \& Macrae, C. N. (2009). The Rhythm of Rapport: Interpersonal Synchrony and Social Perception. Journal of Experimental Social Psychology, 45, 585-589. https://doi.org/10.1016/j.jesp.2009.02.002

Mitkidis, P., McGraw, J. J., Roepstorff, A., \& Wallot, S. (2015). Building Trust: Heart Rate Synchrony and Arousal during Joint Action Increased by Public Goods Game. Physiology and Behavior, 149, 101-106. https://doi.org/10.1016/j.physbeh.2015.05.033

Nagaoka, C., \& Komori, M. (2008). Body Movement Synchrony in Psychotherapeutic Counseling: A Study Using the Video-Based Quantification Method. IEICE Transactions on Information and Systems, 91, 1634-1640. https://doi.org/10.1093/ietisy/e91-d.6.1634

Nagaoka, C., Yoshikawa, S., \& Komori, M. (2006). Embodied Synchrony of Nonverbal Behaviour in Counselling: A Case Study of Role Playing School Counselling. In The 28th Annual Conference of the Cognitive Science Society (pp. 1862-1867). Vancouver: Psychology Press.

Orsucci, F., Giuliani, A., Webber, C. L., Zbilut, J. P., Fonagy, P., \& Mazza, M. (2006). 
Combinatorics and Synchronization in Natural Semiotics. Physica A: Statistical Mechanics and Its Applications, 361, 665-676. https://doi.org/10.1016/j.physa.2005.06.044

Paxton, A., \& Dale, R. (2012). Frame-Differencing Methods for Measuring Bodily Synchrony in Conversation. Behavior Research Methods, 45, 329-343. https://doi.org/10.3758/s13428-012-0249-2

Ramseyer, F., \& Tschacher, W. (2006). Synchrony: A Core Concept for a Constructivist Approach to Psychotherapy. Constructivism in the Human Sciences, 11, 150-171.

Ramseyer, F., \& Tschacher, W. (2011). Nonverbal Synchrony in Psychotherapy: Coordinated Body Movement Reflects Relationship Quality and Outcome. Journal of Consulting and Clinical Psychology, 79, 284-295. https://doi.org/10.1037/a0023419

Ramseyer, F., \& Tschacher, W. (2014). Nonverbal Synchrony of Head- and Body-Movement in Psychotherapy: Different Signals Have Different Associations with Outcome. Frontiers in Psychology, 5, 1-9. https://doi.org/10.3389/fpsyg.2014.00979

Reich, C. M., Berman, J. S., \& Dale, R. (2014). Vocal Synchrony in Psychotherapy. Journal of Social and Clinical Psychology, 33, 481-494.

https://doi.org/10.1521/jscp.2014.33.5.481

Richardson, M. J., \& Chemero, A. (2014). Complex Dynamical Systems and Embodiment. In The Routledge Handbook of Embodied Cognition (pp. 39-50). Abingdon: Routledge.

Sales, C. M. D., \& Alves, P. C. G. (2016). Patient-Centered Assessment in Psychotherapy: A Review of Individualized Tools. Clinical Psychology: Science and Practice, 23, 265-283. https://doi.org/10.1111/cpsp.12162

Schmidt, R. C., \& Richardson, M. J. (2008). Dynamics of Interpersonal Coordination. In A. Fuchs, \& V. K. Jirsa (Eds.), Coordination: Neural, Behavioral and Social Dynamicsdynamics (pp. 281-308). Berlin: Springer. https://doi.org/10.1007/978-3-540-74479-5_14

Shockley, K. D. (2005). Cross Recurrence Quantification of Interpersonal Postural Activity. In M. Riley, \& G. Van Orden (Eds.), Tutorials in Contemporary Nonlinear Methods for the Behavioral Sciences (pp. 142-177). USA: National Science Foundation.

Shockley, K. D., \& Riley, M. A. (2015). Interpersonal Couplings in Human Interactions. In C. L. Webber, \& N. Marwan (Eds.), Recurrence Quantification Analysis Theory and Best Practices (pp. 399-421). Berlin: Springer.

https://doi.org/10.1007/978-3-319-07155-8_14

Sleimen-Malkoun, R., Temprado, J., \& Hong, S. L. (2014). Aging Induced Loss of Complexity and Dedifferentiation: Consequences for Coordination Dynamics within and between Brain, Muscular and Behavioral Levels. Frontiers in Aging Neuroscience, 6, 1-17. https://doi.org/10.3389/fnagi.2014.00140

Tognoli, E., Lagarde, J., DeGuzman, G. C., \& Kelso, J. A. S. (2007). The Phi Complex as a Neuromarker of Human Social Coordination. Proceedings of the National Academy of Sciences of the United States of America, 104, 8190-8195. https://doi.org/10.1073/pnas.0611453104

Tschacher, W., \& Bergomi, C. (2011). The Implications of Embodiment. Imprint Academic.

Tschacher, W., \& Pfammatter, M. (2016). Embodiment in Psychotherapy-A Necessary Complement to the Canon of Common Factors? In European Psychotherapy 2016/ 2017 (pp. 5-21). Norderstedt: Books on Demand.

Vicaria, I. M., \& Dickens, L. (2016). Meta-Analyses of the Intra- and Interpersonal Outcomes of Interpersonal Coordination. Journal of Nonverbal Behavior, 40, 335-361. 
https://doi.org/10.1007/s10919-016-0238-8

Wachsmuth, I., Lenzen, M., \& Knoblich, G. (2008). Embodied Communication in Humans and Machines. Oxford: Oxford University Press. https://doi.org/10.1093/acprof:oso/9780199231751.001.0001

Wallot, S., Fusaroli, R., Tylén, K., \& Jegindø, E. M. (2013). Using Complexity Metrics with R-R Intervals and BPM Heart Rate Measures. Frontiers in Physiology, 4, 1-8. https://doi.org/10.3389/fphys.2013.00211

Wallot, S., Mitkidis, P., McGraw, J. J., \& Roepstorff, A. (2016). Beyond Synchrony: Joint Action in a Complex Production Task Reveals Beneficial Effects of Decreased Interpersonal Synchrony. PLOS ONE, 11, e0168306. https://doi.org/10.1371/journal.pone.0168306

Webber, C. L., \& Zbilut, J. P. (2005). Recurrence Quantification Analysis of Nonlinear Dynamical Systems. In M. Riley, \& G. Van Orden (Eds.), Tutorials in Contemporary Nonlinear Methods for the Behavioral Sciences (pp. 26-94). USA: National Science Foundation.

Wiltermuth, S. S., \& Heath, C. (2008). Synchrony and Cooperation. Psychological Science, 20, 1-5. https://doi.org/10.1111/j.1467-9280.2008.02253.x

Zbilut, J. P., \& Webber, C. L. (1992). Embeddings and Delays as Derived from Quantification of Recurrence Plots. Physics Letters A, 171, 199-203. https://doi.org/10.1016/0375-9601(92)90426-M

Zbilut, J. P., Giuliani, A., \& Webber, C. L. (1998). Detecting Deterministic Signals in Exceptionally Noisy Environments Using Cross-Recurrence Quantification. Physics Letters A, 246, 122-128. https://doi.org/10.1016/S0375-9601(98)00457-5 